\title{
Bilateral synchronous benign ovarian neoplasm: A rare occurrence
}

\author{
I U Takai, ${ }^{1}$ MBBS, MHPM, FCAI, FMCOG; U A Umar, ${ }^{1}$ MD, FWACS; H A Nggada, ${ }^{2}$ MBBS, FMC Path; $\mathbf{M}$ Bukar, ${ }^{3}$ MBBS, \\ MHPM, FMCOG, FWACS; B M Audu, ${ }^{3}$ MBBS, MSc, FMCOG
}

${ }^{1}$ Department of Obstetrics and Gynaecology, Bayero University/Aminu Kano Teaching Hospital, Kano, Kano State, Nigeria

${ }^{2}$ Department of Histopathology, University of Maiduguri Teaching Hospital, Maiduguri, Borno State, Nigeria

${ }^{3}$ Department of Obstetrics and Gynaecology, University of Maiduguri Teaching Hospital, Maiduguri, Borno State, Nigeria

Corresponding author: I U Takai (takaiidris@yahoo.co.uk)

Bilateral synchronous ovarian tumours are defined as the occurrence of two or more histologically distinct tumours in the ovaries. Synchronous tumours of the female genital tract are rare and the association of mature cystic teratoma with contralateral serous cystadenoma is uncommon. We report the rare occurrence of a giant mature cystic teratoma with a coexisting contralateral serous cystadenoma in a 32-year-old para 5. The patient refused an initial offer for surgery 10 years earlier, and had three successive term pregnancies and deliveries, with the tumour intact. She later had bilateral salpingo-oophorectomy of a $55 \mathrm{~kg}$ left ovarian mass and a $2 \mathrm{~kg}$ right ovarian mass, which revealed a left ovarian benign cystic teratoma and a right ovarian papillary serous cystadenoma at histology. Neglected ovarian tumours are still encountered in sub-Saharan Africa and may involve diagnostic and management challenges. There is a need to educate women and the community on ovarian neoplasms and the need to present early for effective management. Women's reproductive health rights need to be encouraged and possibly legislated in our setting.

S Afr J Obstet Gynaecol 2017;23(1):31-34. DOI:10.7196/SAJOG.2017.v23i1.1130

Synchronous tumours of the female genital tract, which are most often ovarian and endometrial, ${ }^{[1]}$ are rare and account for only $0.7-1.8 \%$ of all tumours. ${ }^{[2]}$ The association of mature cystic teratoma with contralateral serous cystadenoma is uncommon. The most common benign germ cell tumours (GCTs) of the ovary are the mature cystic teratomas (MCTs). Teratomas comprise about 15 - 20\% of all ovarian tumours. ${ }^{[3]}$ They are composed of one or more germ layers derived from a pluripotent precursor cell. MCTs are frequently multicystic and contain sebaceous fluid, hair, teeth, bone or skin, and are bilateral in $10-12 \%$ of cases.

Serous cystadenoma is also a benign tumour of the ovary, which is responsible for about $30 \%$ of all epithelial ovarian neoplasms. They are commonly unilocular, containing clear yellow fluid, and about $10-15 \%$ are bilateral. ${ }^{[4]}$ The association of MCT with contralateral serous cystadenoma is uncommon.

\section{Case report}

We present a case of bilateral synchronous benign ovarian neoplasm that comprised a giant teratoma weighing $55 \mathrm{~kg}$, with a coexisting contralateral serous cystadenoma that weighed $2 \mathrm{~kg}$. The patient was a 32-year-old para 5 who had last given birth 3 years prior to presentation at the Gynaecology Clinic of the State Specialist Hospital, Maiduguri, Nigeria. She was referred from a private clinic. Her problem started $\sim 10$ years prior to presentation with abdominal swelling, when an ultrasound scan revealed a complex left ovarian cyst. She was counselled for surgery but defaulted. She had tapping of the swelling in some of the hospitals she visited and had previously been on treatment with traditional medicine. Despite recurrent swelling, she had three successive term pregnancies and deliveries. At the time of presentation at our facility, the patient believed that she was carrying a twin pregnancy and mentioned she had been informed by traditional healers that her condition was not suitable for surgery. As such, she was fearful of dying during the removal of the mass. She had also been threatened with divorce if she accepted any form of surgical intervention. These factors had made the patient refuse any surgical intervention at an earlier stage.

In the year prior to her presentation at the referring hospital, the abdominal swelling increased rapidly and was associated with respiratory and abdominal discomfort, which necessitated her presentation at the hospital. Occasional abdominal pain and vomiting was accompanied by a loss of appetite but there was no abnormal vaginal bleeding or urinary symptoms. She had no body itching, yellowness of the eyes, cough or chest pain, but had associated dyspnoea and weight loss. She had attained menarche at the age of 15 years and had a regular menstrual pattern. All her previous pregnancies were unsupervised and all deliveries were at home. There was no family history of breast, ovarian or colonic cancers and the patient was neither diabetic nor hypertensive.

On examination, she was chronically ill-looking. She weighed $96 \mathrm{~kg}$ and was $1.56 \mathrm{~m}$ tall. Her chest was clinically clear and her cardiovascular system was stable. An abdominal examination revealed a grossly distended abdomen with distended subcutaneous veins and a uniformly enlarged mass compatible with a term gestation. The mass was firm to hard, not mobile, smooth and non-tender. The liver, spleen and kidneys were not palpable. There was no demonstrable ascites and bowel sounds were present. A pelvic examination revealed a normal vulva and vagina. However, the cervix was flushed and the uterus could not be delineated. Both adnexae were full. The pouch of Douglas was free and the rectal examination was normal. A working diagnosis of huge ovarian tumour was made (Fig. 1A).

Her full blood count result was normal with a packed cell volume of $31 \%$. She had normal electrolyte, urea and creatinine levels and 


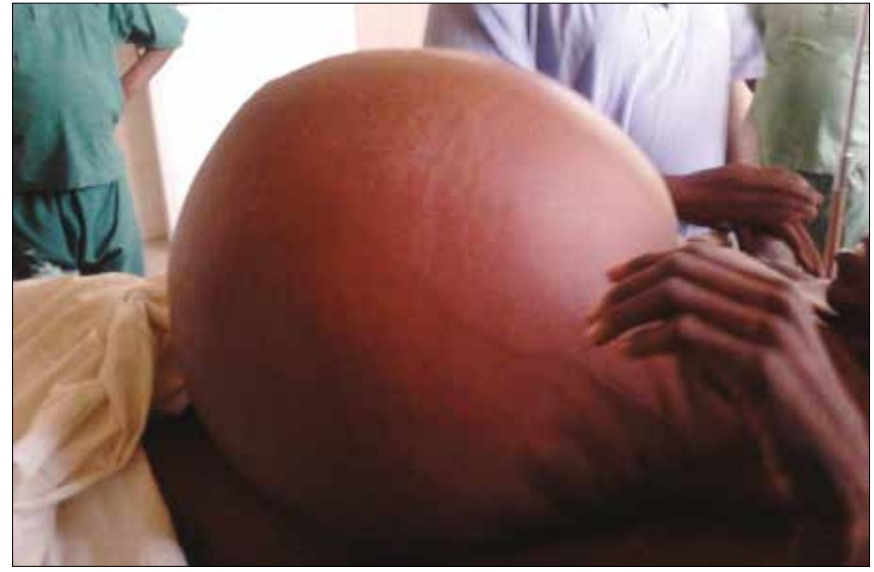

Fig. 1A. Grossly distended abdomen and veins in a patient with ovarian tumour.

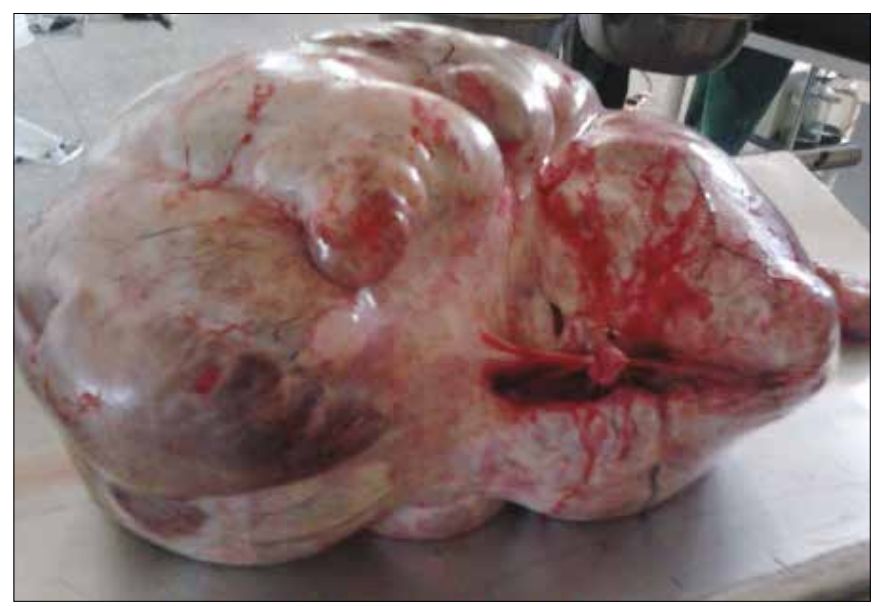

Fig. 1B. Gross appearance of left ovarian benign cystic teratoma weighing $55 \mathrm{~kg}$ (ventral view).

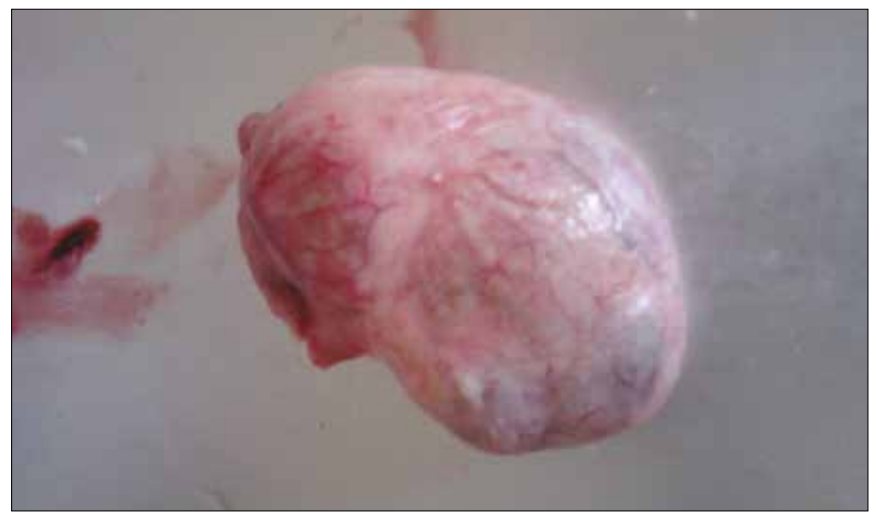

Fig. 1C. Gross appearance of right ovarian papillary serous cystadenoma weighing $2 \mathrm{~kg}$.

tested negative for HIV and the hepatitis B surface antigen (HBsAg). The patient's urinalysis, plain chest X-ray, fasting blood sugar and liver function tests were all normal. A urinary pregnancy test was negative and an abdomino-pelvic ultrasound scan revealed a huge multiseptated, cystic intra-abdominal mass arising from the pelvis, with mixed echogenicity and a thick wall. Measurements of the mass were beyond the capacity of the probe. The liver and spleen appeared normal but the kidneys had dilated calyxes. The uterus was not visualised. She was counselled for exploratory laparotomy and consented on condition that her uterus be preserved. Findings at surgery were minimal ascites and a massive twisted left ovarian cystic mass that adhered to the anterior abdominal wall, measuring $62 \mathrm{~cm}$ at its widest diameter, and weighing $\sim 55 \mathrm{~kg}$ with a thick smooth wall without excrescences on the surface (Fig. 1B). The right ovary was also found to be cystic, measuring $11 \mathrm{~cm}$ at its widest diameter and weighing $\sim 2 \mathrm{~kg}$ with a thin smooth wall without excrescences on the surface (Fig. 1C). The uterus, tubes, omentum, liver and spleen were normal. The bowels were free and the pouch of Douglas was empty. The pelvis appeared generally clean and the patient had bilateral salpingo-oophorectomy. Macroscopic opening of the left ovary revealed a multilocular cystic cavity containing gelatinous material and a smooth inner lining. A focal solid area, hair shafts and cartilage were noted. Sections of the left ovary showed triphasic elements composed of skin, intestinal epithelium, cartilage, adipose and neural tissue, as well as lymphoid cells, but there was no evidence of malignancy (Fig. 2). Opening of the right ovary revealed a multilocular cyst containing about $800 \mathrm{~mL}$ of blood-tinged serous fluid. Sections of the right ovary showed a fibro-collagenous cyst wall lined by a single layer of cuboidal epithelium with focal areas of papillary infolding and haemorrhage consistent with serous cystadenoma (Fig. 3). The ascitic fluid did not show any malignancy

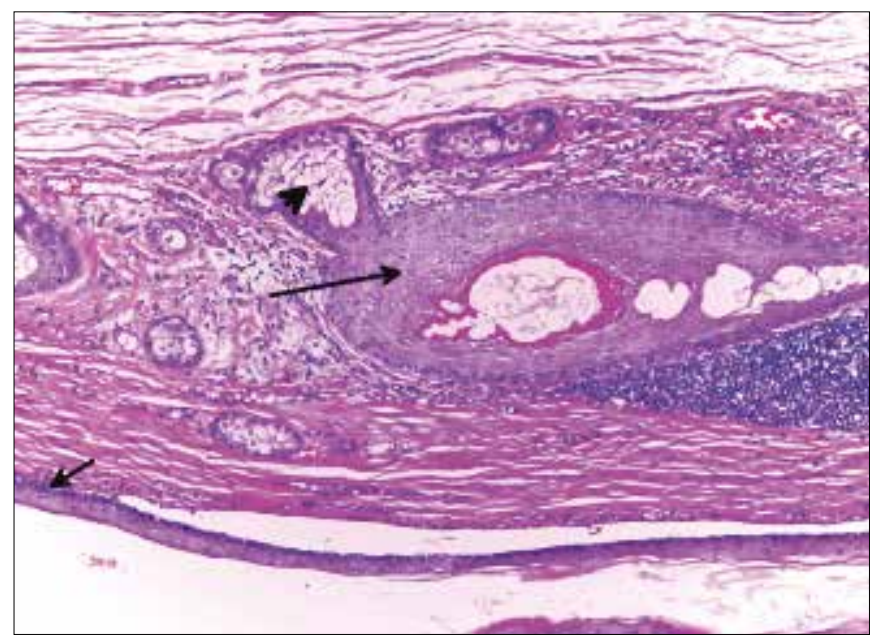

Fig. 2. HE stain of a section of the matured left ovarian benign cystic teratoma showing matured cartilage (long arrow), respiratory epithelium (short arrow) and adipose tissue (arrow head).

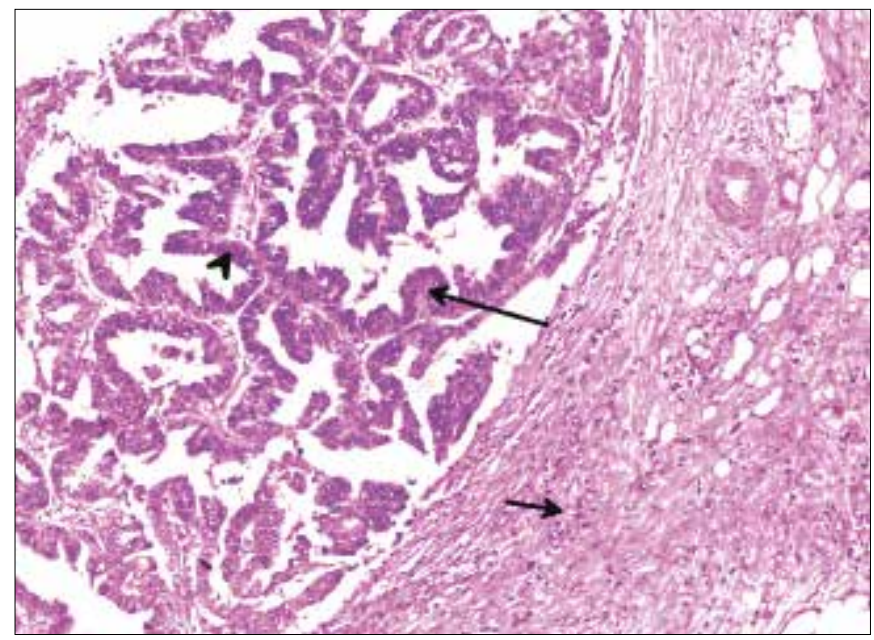

Fig. 3. HE stain of a section of the right ovarian papillary serous cystadenoma showing cuboidal epithelium (arrow head), papillary projections (long arrow) and connective tissue stroma (short arrow). 
and the final diagnosis was a left ovarian benign cystic teratoma and right ovarian papillary serous cystadenoma. She had an uneventful postoperative period and was discharged. At a follow-up visit to a gynaecology clinic 3 weeks later, she was clinically stable and the patient was set to be followed up for life.

\section{Discussion}

The term synchronous tumour is used when two or more tumours occur simultaneously in a patient. Synchronous tumours of the female genital tract are rare, accounting for $0.7-1.8 \%$ of tumours in the female genital tract. ${ }^{[2]}$ The most frequently encountered synchronous tumours of the female genital tract are ovarian (as in this case) and endometrial. ${ }^{[1]}$ Although most are double, triple synchronous tumours have been reported. ${ }^{[2]}$ Pathological screening of 957 benign cystic teratomas yielded nine multiple ipsilateral ovarian teratomas. ${ }^{[5,6]}$ Serous tumours rarely occur in combination with GCTs. ${ }^{[7]}$ Common combinations with ovarian tumours include mucinous cystadenoma and a combination of Brenner tumour, mature cystic teratoma, Sertoli-Leidig cell tumour may be seen. ${ }^{[8]}$

Ovarian tumours are currently classified based on the histogenesis of the ovary. Early development of the ovary is characterised by segregation and migration of the primordial germ cells from their sites of origin to the genital ridges (bilateral thickenings of the coelomic epithelium). This is followed by the proliferation of the coelomic epithelium and underlying mesenchyme. The ovary is then divided into the peripheral cortex and central medulla. The cortex develops further, while the medulla undergoes involution. ${ }^{[9]}$ The World Health Organization histological classification of ovarian tumours separates ovarian neoplasms according to the most probable tissue of origin: surface epithelium (65\%), germ cells (15\%), sex-cord stromal tissue (10\%), miscellaneous (5\%) and tumours of metastatic origin $(10 \%) .{ }^{[10]}$

Teratomas are the most common GCTs of the ovary. They are composed of multiple cell types derived from one or more of the three germ layers. Mature teratomas are benign cystic teratomas. They occur mainly in women of reproductive age, as in our patient, but may occur in postmenopausal women and in children. These tumours may contain mature tissue of ectodermal (skin, brain), mesodermal (muscle, fat) and endodermal (mucinous or ciliated epithelium) origin. ${ }^{[1]}$

Serous tumours, of which $50 \%$ are usually seen in women $<40$ years of age, ${ }^{[12]}$ develop by invagination of the surface epithelium of the ovary and secrete serous fluid. Serous tumours are generally benign; 5 - 10\% have borderline malignant potential, and $20-25 \%$ are malignant. Serous cystadenomas usually are multilocular and sometimes have papillary projections. ${ }^{[12]}$ Giant ovarian tumours are becoming rare in current medical practice especially in developed countries where most cases are discovered early during routine check-ups or ultrasonography. However, in developing nations such as Nigeria where access to healthcare is poor, coupled with a low literacy level, neglected giant ovarian tumours are still encountered. In our case, the patient had a low literacy level and had been threatened with divorce, which led to an initial delay in intervention. Giant ovarian teratomas commonly present with acute abdominal pain caused by adnexal torsion and abdominal distension due to tumour growth. Some patients, as in ours, may also have nonspecific abdominal complaints from pressure effects of the mass such as dyspnoea, dyspepsia and anorexia. ${ }^{[13]}$
A review of the literature revealed that giant ovarian tumours were reported in some studies more than 100 years ago. In 1905, Spohn ${ }^{[14]}$ reported on a 43-year-old woman with a simple abdominal cyst that was drained preoperatively over 7 days, yielding $\sim 114 \mathrm{~L}$ of gelatinous fluid. The tumour was estimated to be $146 \mathrm{~kg}^{[15,16]}$ In 1994, O'Hanlan ${ }^{[15]}$ removed the largest known cystic tumour, weighing $137.4 \mathrm{~kg}$. Madhu et al. ${ }^{[16]}$ recently reported a complete resection of a giant ovarian tumour weighing $57 \mathrm{~kg}$, similar to our patient. To our knowledge, our patient had the largest tumour ( $55 \mathrm{~kg}$ ) ever reported in Nigeria.

Estimation of tumour markers such as CA125 and imaging evaluation such as magnetic resonance imaging and computed tomography were indicated in this patient; however, these were not performed due to non-availability in the hospital and the financial constraints associated with referral to other health centres. Although some giant cystic teratomas are amenable to laparoscopic removal after decompression, this is not possible in solid or giant tumours. Our patient was initially counselled for hysterectomy and bilateral salpingo-oophorectomy for fear of malignancy, but did not consent to the former. However, conservative surgery such as ovarian cystectomy and salpingo-oophorectomy is adequate for benign tumours of the ovary. ${ }^{[17]}$ The incidence of malignant transformation in ovarian teratomas is $1-2 \%$, and the malignant component may stimulate a separate ovarian mass. ${ }^{[18]}$ Fortunately for this patient there were no histological signs of malignancy.

This case attracted our attention for at least three reasons: firstly it was a synchronous tumour which occurred with a GCT and secondly, it was huge. This tumour was also among the largest ever reported in Nigeria. The massive dimension can be attributed to a delay in seeking care, superstitious beliefs, fear of divorce and dying from surgical intervention.

\section{Conclusion}

Neglected ovarian tumours are still encountered in sub-Saharan Africa and may involve diagnostic and management challenges. Owing to poverty and illiteracy, patients in rural settings often seek medical advice at a very late stage in disease progression. Our patient delayed surgical intervention for 10 years before finally consenting to have the tumour removed. Had the tumour been malignant, she would have presented terminally ill with advanced disease or she may have died from its complications. There is a need to educate women and their communities on ovarian neoplasms and the need to present early for effective management.

\footnotetext{
1. Tong SY, Lee YS, Park JS, Bae SN, Lee JM, Namkoong SE. Clinical analysis of synchronous primary neoplasms of the female reproductive tract. Eur J Obstet Gynecol Reprod Biol 2008;136(1):78-82. https://doi.org/10.1016/j.ejogrb.2006.09.010

2. Dudnikova A, Veriozkey I, Pete I. Synchronous tumours of female genital tract: Triple malignant and one benign tumour. Hung Onkol 2012;56(1)55-59.

3. Shanmughapriya S, Senthil Kumar G, Balakrishnan K, Vasanthi N, Vinodhini K, Natarajaseenivasan K. Bilateral ovarian teratoma complicated with carcinosarcoma in a 68 year old woman: A case report. BMC Cancer 2011;11(1):218. https://doi.org/10.1186/1471-2407-11-218

4. Oliver D, Vicki VB. Premalignant and malignant diseases of the ovaries and oviductcts. In: Decherney AH, ed. Current Obstetrics and Gynecologic Diagnosis and Treatment. 9th ed. New York: Lange Medical Books, 2003:933-958.

5. Samuel CJ, George LJ. Sonographic diagnosis of multiple unilateral teratomas. J Ultrasound Med 2001;20(3):279-281. https://doi.org/10.7863/jum.2001.20.3.279

6. Damewood M, Rosenshein NB, Woodruff JD. Multiple benign cystic teratomas of the ovary. Diag Gynecol Obstet 1982;4(3):243-245.

7. Bachhav AA. Serous cystadenoma with co-existing stromal tumour with sex-cord stromal elements: An extremely rare tumour. Open J Obstet Gynecol 2014;4(3):105-108. https://doi.org/10.4236 ojog.2014.43019

8. Jayalakshmy PS, Poothiode U, Krishna G, Jayalakshmy PL. Ovarian fibroma with serous cystadenoma - an unusual combination: A case report. Case Rep Obstet Gynecol 2012;2012:1-3. https://doi org/10.1155/2012/641085

9. DiSaia PJ, Creasman WT. Epithelial ovarian cancers. In: Disala ED, Creasman WT, eds. Clinical
} Gynecologic Oncology, 4th ed. Saint Louis: Mosby, 1993:333-334. 


\section{CASE REPORT}

10. Serov SF, Scully RE, Sobin, LH. Histological Typing of Ovarian Tumours. Geneva: WHO, 1973:17-18

11. Outwater EK, Siegelman ES, Hunt JL. Ovarian teratomas: Tumor types and imaging characteristics. RadioGraphics 2001;21(2):475-490. https://doi.org/10.1148/radiographics.21.2.g01mr09475

12. Dey M, Pathak N. Giant serous papillary cystadenoma. Med J Armed Forces India 2011;67(3):272273. https://doi.org/10.1016/s0377-1237(11)60059-2

13. Ye L-Y, Wang, J-J, Liu D-R, Ding G-P, Cao L-P. Management of giant ovarian teratoma: A case series and review of the literature. Oncol Lett 2012;4(4):672-676. https://doi.org/10.3892/ol.2012.793

14. Spohn AE. Multicystic ovarian tumour weighing 328 pounds. Texas Med J 1905:1273-1274.
15. O'Hanlan KA. Resection of a 303.2-pound ovarian tumor. Gynecol Oncol 1994;54(3):365-371. https://doi.org/10.1006/gyno.1994.1225

16. Madhu YC, Harish K, Gotam P. Complete resection of a giant ovarian tumour. Gynecol Oncol Case Rep 2013;6:4-6. https://doi.org/10.1016/j.gynor.2013.05.001

17. Kamel RM. A massive ovarian mucinous cystadenoma: A case report. Reprod Biol Endocrinol 2010;8(1):24. https://doi.org/10.1186/1477-7827-8-24

18. Ahmed SE, Ayat SAM. Multiple bilateral huge synchronous ovarian mature cystic teratomas: A rarely encountered condition in practice. Egypt J Radiol Nucl Med 2014;46(1):195-197. http://doi. org/10.1016/j.ejrnm.2014.11.001 